August 2008, Volume 52, Issue 3, pp 465-488

\title{
A Multi-Modal haptic interface for Virtual Reality and Robotics
}

\author{
Michele Folgheraiter, Giuseppina Gini, Dario Vercesi
}

21st June 2006

\begin{abstract}
In this paper we present an innovative haptic device that combines the electro-tactile stimulation with the force and visual feedbacks in order to improve the perception of a virtual world. We discuss about the sensation evoked in a user by the haptic, force, and visual interface provided by this device, implemented as a special glove, equipped with sensors and actuators connected to a PC. The techniques used to recreate tactile and kinesthetic sensations are based on an innovative use of cutaneous stimulation integrated with actuators and 3D modelling techniques. We discuss about the specificity of haptic interfaces, their controllers, their open problems. We present results about generating the sensation of touching virtual objects with our device. Experiments show also that, using a multi-modal sensorial pattern of stimulation, the subject perceives more realistically the virtual object. We discuss about possible use of the same technique as a way to interface intelligent robots.
\end{abstract}

Keywords Virtual Reality, Electro-tactile Stimulation, Haptic Devices, Robotic Grasp, Haptic display

\section{Introduction}

Haptic sense is very important for human beings. Together with vision, the touch holds a primary role, especially during activities like objects manipulation. Sometime touch can also overcome or substitute vision, as in the case of blind people [1] that are able to acquire the properties of an object only touching and lifting it. Indeed with the haptic sense it is possible to gain plenty of information about weight, shape, texture, temperature, consistency. This means that if we want improve the human interaction with a virtual world we have to take in account the properties and modality of this fundamental sensorial channel.

There are many ways to evoke a touch sensation, nevertheless we can make a first classification in two methods that:

- reproduce the physical stimulus

- stimulate directly the receptor's nerve 
Even if the purpose is the same, there is a conceptual difference between these two methods. In the first case, when reproducing the physical stimulus we act directly on the receptor body; this causes the depolarization of the membrane and therefore an action potential is sent to the central nervous system (CNS). Using the second approach we directly depolarize the receptor's membrane, injecting a current inside the tissue. This again causes the action potential, but without generating the physical stimulus. We can say that this methodology uses a more direct interface with the nervous system.

It is possible to reproduce the touch stimulus using different technologies. Researchers at Tokyo University of Agriculture and Technology [2] built a tactile display that uses ferro-magnetic chips attached directly to the skin; the pressure applied is proportional to the magnetic field generated by coils located near the chips. Using an array of 4X4 pneumatic orifices, the touch display designed at University of Salford [3] can emulate the object shape and texture. The airjet pressure of each single pin is modulated in order to change the force that acts on the user fingertip. The system can perform a wide range of statical and dynamical tactile sensations with a virtual or remote environment. Another way to generate the tactile stimulus has been proposed by T. Yoshikawa et al [4]; in their haptic device the problem of permanent contact between finger and device is solved introducing a finger tracking system. When the user moves the finger around the free work space, the haptic device moves with it avoiding any contact with the finger. When a virtual object is encountered (in the correspondent virtual environment) the contact between the device and the finger is allowed and a reaction force is transmitted to the user. In this case, due to the device framework, more importance is given to the kinesthetic feedback than the touch feedback

A complete different way to generate the touch stimulus is the method that uses a current to depolarize the receptor's membrane. This is also referred as electro-tactile stimulation; using a superficial electrode interfaced with the user skin, and a high voltage $(30-300 \mathrm{~V})$ source, it is possible inject a biphasic current inside the tissue. Different experiments was done to find the best pattern of stimulation; Kaczmarek et al in their early studies [5] discovered that the static skin-electrode resistance decreases non-linearly with increasing stimulation current. This suggests to model the skin-electrode interface with a resistancecapacitor (RC) circuit. In a successive work the authors [6] experimented a complex electro-tactile display; the haptic device consists in a matrix of $7 \mathrm{X} 7$ small electrodes that fits the size of a medium fingertip. Activating independently the electrodes and modulating the current it is possible to generate complex patterns of stimulation. They discovered that the number of patterns correctly recognized increases monotonically with the current intensity of the stimulation.

In their work Hiroyuki Kajimoto et al [7] demonstrated how it is possible to use an electro-tactile display to augment the human perception. Their system is composed of two principal parts: the sensory layer that translates optical information in electrical signal, and the electrode layer that converts the information transduced by the sensory layer in a proper touch stimulus. The electrode layer is composed by a matrix of $4 \mathrm{X} 4$ electrodes that are governed by a time divi- 
August 2008, Volume 52, Issue 3, pp 465-488

sion scanning, so when an electrode is active the others are connected to the ground. In this way a single high voltage generator is used in turn for each single electrode.

Another interesting discovery is reported in [8], about the different perception of the anodic current versus the cathodic current. In the first case the sensation perceived by the user is similar to a vibration, instead, in the other case, the feeling is similar to a pressure.

Other studies were done to improve the current regulation of the high voltage impulse generators [9] or to optimize the power delivered on the base of the impulse frequency [10], with the purpose of reducing the electrodes area and increasing the dynamic range of the haptic device.

The use of this kind of haptic rendering is quite different from the well known methods developed with the PHANToM device [11]. While for Phantom the tactile exploration of the world is similar to getting information from a pencil hold in the hand and used as a probe, our glove could in principle give tactile information in more points of the hand. However in this study we use only one finger. The multi contact and multi body dynamics of an haptic device have been already developed for PHANToM, for instance in [12].

A more recent use of haptic devices is in the field of robotics and humanoid robotics in particular. Here the problem is to program or to remotely control a robot to make complex manipulation tasks.

A first example of the use of an haptic device in robotics is in teaching how to grasp an object. Given an object with a certain shape and mechanical properties, the reaching problem is how to approach the object, the grasping problem is how to perform the grasp. To do it the robot usually receives the position and orientation of the object, and computes the best hand configuration and the minimum force needed for the grasp. Of course the object static and dynamic parameters are very important in order to plan the grasp; this task requires solving complicate Kinematic and Dynamic problems. A good grasp should be stable, which means that the object should not move or rotate relatively to the hand. The energy spent by the hand's actuators should be as low as possible, and this depends strictly on the contacts points of the fingers with the object.

A manner to overcome these issues is to teach the robot "how" to perform the grasp after learning from human executions. The glove we developed is equipped with a set of sensors that can measure the position of the phalanxes and the force applied by the thumb and the index during the grasp. We conducted a series of data acquisition on objects of difference dimensions and materials, the data was recorded, normalized and then used to train a neural network (NN)able to generalize grasps for objects never used during the learning process. In order to use the outputs generated by the NN to control an artificial hand, it is necessary to scale them using the kinematic and dynamic models of the manipulation device. We developed these models for our prototype of artificial hand "WhiteFingers". In particular from the static model we converted the force on the fingertip to the needed moments on the finger joints. This work is reported in [13].

In the next Section we will discuss more about the open problems of haptic 
control. In section 3 we describe, in detail, the physiology of the skin and the characteristics of the receptors principally involved in measuring the mechanical interaction between the manipulated object and the hand. In section 4 we describe the design principles of our haptic interface and the global architecture. Finally in section 5 we describe its use through the answers of a set of voluntary people.

\section{Haptics: open problems}

Haptic is the discipline that study the tactile sensations and how these can be reproduced by an artificial system. The term originates from the Greek word "haptikos" that means "about touching".

The main topics in haptics are: structuring data for the model of the world, collision detection, contact point following. These issues are not exceptionally new or challenging, as most of the research has been already done for computer graphics. There are some subtle differences, but the main concepts and solutions can be reused. Some problems are new, as computation of the force direction and amplitude of the contact force, force shading, friction and haptic texture, stability. These problems have been tackled and solved individually [14][15][16] but there is no documented attempt to bring all of them together.

When a user interacts with a virtual world through a simulator, the overall effect of immersion is crucial. The simulation should use models that are as close as possible to accurate models of contact and friction. Obviously the physical limitations of the interface must be taken into account when designing the simulation, but the simulation should not be developed with any one haptic device as unique target. The simulation should nonetheless avoid models that generate phenomenons that cannot be realized by the interfaces. For this reason the designers of a good simulation cannot ignore the capabilities of the hardware.

This situation shows clearly the immaturity of haptics, because a well engineered framework should separate clearly the stages it is composed of, and it does not happen at present. Moreover the goals of two stages are in conflict because the simulation should have as high a bandwidth as possible, while the haptic device must limit it for stability, a critical issue that appears almost everywhere in haptics.

To make this point clear let's consider an example: an application that makes extensive use of graphics is built having in mind clearly what are the capabilities of the hardware, but if a user does not own the latest graphic card the quality of the output will be very poor. Nevertheless the application will not crash, nor will it misbehave. With an haptic application the limitations of the hardware often cause a wrong behavior. This is due to the fact that the feedback loops that go through haptic and graphic interfaces are very different. This is why haptics draws a lot of attention by people involved in control. The closed loop effects that are ignored for classical loops connecting: virtual environment, video, user, input device must be analyzed and understood in haptics, as they play a major role in the stability of the system. 
The interaction of physical systems obeys a set of underlying laws, while the interaction of virtual systems obeys similar laws only approximately. The consequences of approximate obedience can be profound. For instance, in the physical world we could scarcely even contemplate the possibility that, upon bolting together two steel beams, the entire assembly would exhibit growing oscillations. But this is precisely what might occur in a virtual world if appropriate laws are not enforced to govern interaction. To ensure stable interactive behavior the physical world relies heavily upon the property of passivity. It is well-known that the coupling of passive systems is guaranteed to be stable. It is by no means established that virtual worlds must rely on passivity, but surely some comparable underlying property is essential for stability. Haptic display makes the need for stability more acute for two reasons. The first is that the human tactile sensory apparatus is extremely receptive to small amplitude mechanical vibrations in the $100 \mathrm{~Hz}-1 \mathrm{kHz}$ range (while vision is not). The second is that the human is also a dynamic system. Thus, even though a non-passive virtual environment may be stable, interaction with a human via a haptic interface may cause instability. This topic will be only partially approached through a study of the sensation felt by the user; we will see how sensations are fine and timely, and how the visual feedback is used.

\section{Skin physiology from a functional point of view}

The receptors are skin organs that permit to perceive physical quantity internally or externally of our body.

In particular we are interested in the physiology of the mechanoreceptors, the receptors that transduce mechanical stimuli in nervous signals. The mechanism of transduction is well know; when a pressure deforms the receptor's membrane some ionic channels will open, and the ions $\left(\mathrm{Na}^{+}, \mathrm{K}^{+}\right)$cross the channels depolarizing the membrane. If the membrane potential reaches a certain threshold, depending on the receptor characteristics, an action potential is generated and transmitted along the receptor's axon to the primary hand's nerve. In the human skin, at different depths, we can find four kinds of mechanoreceptors specialized in perceiving different mechanical stimulus: Merkel Cell,Meissner corpuscle, Pacinian corpuscle, and Ruffini corpuscle.

Merkel cell: The Merkel cells are sensitive to local stress strain field, therefore are able to detect edges, corners and curvature of the manipulated objects. Their response to a surface or object form is linear with the skin deformation and independent on the force of application. The spatial resolution is about $0.5 \mathrm{~mm}$ and it response is ten time more sensitive to dynamic than to static stimuli [17].

Meissner corpuscle: Meissner corpuscles are insensitive to static skin deformation; their spatial resolution is $(3-5 \mathrm{~mm})$. Principally they are responsible for detecting slippage between the skin and the object. When we lift an object there is frequently a microscopic slip (between the object and the skin) that is perceived by Meissner corpuscles. This information is then sent to the reflex 
circuits of the spinal cord that act in order to increase the grip force and therefore to avoid the slip [18]. These fibers are insensitive to static forces and to very low frequency vibrations; if not, during a gripping the big force exerted would mask the small signal due to slippage.

Pacinian corpuscle: there are about 350 Pacinian receptors for a single finger, connected with a dedicated fiber. Very deeply located in the skin, they are very sensitive and can detect skin movements of about $10 \mathrm{~nm}$. Due to their location they have no spacial resolution; a single Pacinian corpuscle has a receptive field that can cover also the entire hand. These receptors are specialized to detect vibrations that come from the object held in the hand [19]. When we become skilled in using a tool, we are able to perceive the events at the working surface, as the tool behave like and artificial extension of our hand.

Ruffini corpuscle: Ruffini corpuscles are specialized to detect skin stretch. Their receptive fields are five time larger than in case of the Meissner corpuscles, nevertheless they have a smaller spatial resolution. There are two principal roles for these fibers: perception of the direction of the object motion and perception of the hand shape. This second role is very important; with both the muscle spindles and joint afferent, they are able to detect the pattern of the skin stretch. Ruffini corpuscles transmit to the central nervous system (CNS) a neural image of the skin stretch, which is interpreted by the CNS in order to obtain the hand posture. Some studies reveal that the artificial stretch of the skin of the hand produces the illusion of bending the fingers [20]; this effect may be used, in an haptic interface, to evoke a motion perception during the interaction with the virtual object even without really moving the hand.

\section{The haptic device}

The system we designed was intended for two main purposes: as acquisition interface and as haptic interface. In the first application the device is used to acquire the hand posture of a human being performing a grasp. In this case not only the positions of phalanxes are measured, but also the contact forces between the object and the fingers are acquired. The recorded data can be used to move a real robot hand, or to teach through a neural network (NN) how to automatically synthesize the grasp for a given object.

The second use of our system is like an experimental platform to conduct a series of psyco-physical tests on human subjects during the interaction with a virtual object. The main goals is to study the electro-tactile stimulation and the advantages of its integration with others sensorial channels like force feedback and vision.

The hardware consists in: a wearable glove, a sensor board, a electrocutaneous stimulation system, and a PC. On the dorsal part of each phalanxes of the glove are installed angular sensors that detect the relative position of each articulation. In total there are 14 units (figure 1). Force sensors are connected in series with the tendons that transfer the movement from the actuators to the fingertip, these are necessary to measure the force delivered to the user's finger. 


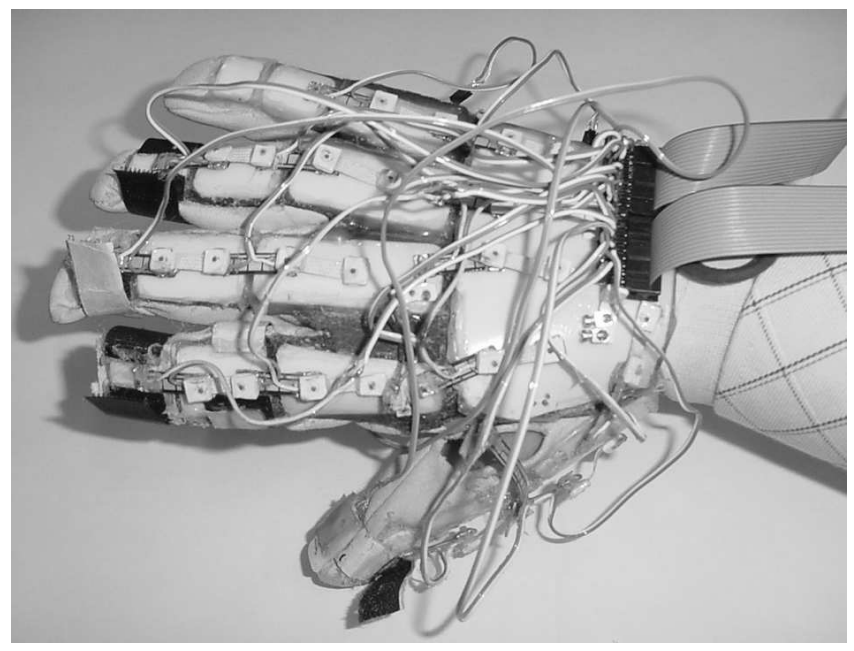

Figure 1: The Glove.

We can recognize three different systems that support the glove: the Force feedback system, the electro-cutaneous stimulation system and the acquisition and control system. Each of these parts have an hardware and a software component. In the following sections we will describe these three systems.

\subsection{The Force feedback system and its connection to the virtual object model}

The force feedback system consists in servo motors with a maximum torque of $10 \mathrm{Kg} / \mathrm{cm}$. They integrates a reduction and sensor system and are directly controllable in position. The movement and the force is transmitted to the fingertip by tendons fixed to the solid plastic bands of the glove (Figure 2). They are configured so that to maximize the component of the servo force that acts perpendicularly to the movement path of the fingertip. The system, according with the virtual object model, delivers the required amount of force.

The reaction force of the virtual object, in first approximation, can be modelled by equation 1

$$
F_{m}(t)= \begin{cases}0 & \text { if } Z_{f t}>Z_{v o} \\ K_{e}\left(\bar{Z}_{v o}-Z_{f t}(t)\right)+K_{d} \dot{Z}_{f t}(t) & \text { if } Z_{f t} \leq Z_{v o}\end{cases}
$$

Where $Z_{f t}$ is the position of the user fingertip and it is calculated from the positions of the phalanxes using the direct kinematic model, $K_{e}$ is the elastic constant, $K_{d}$ is a damping constant and $\bar{Z}_{v o}-Z_{f t}(t)$ is the penetration rate into the object surface. Changing the constants $K_{e}, K_{d}, \bar{Z}_{v o}$ we can emulating object of different dimensions and different materials. For example if $K_{e}$ is very 


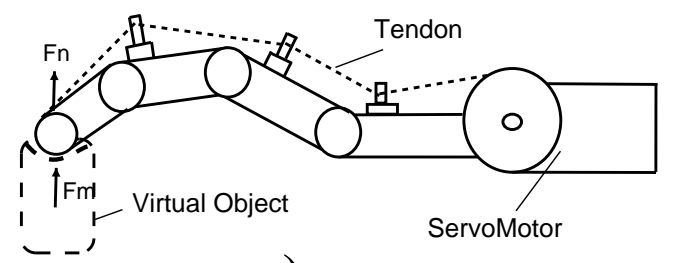

a)

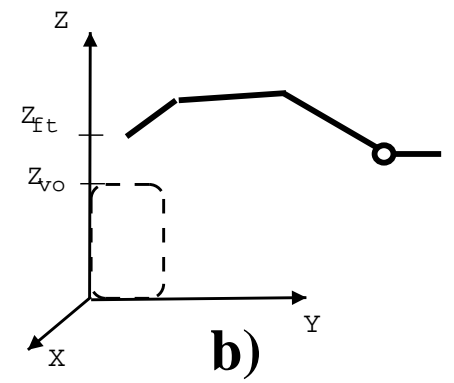

Figure 2: Schema of the artificial tendons.

hight the object will behaves like an hard box, if $K_{e}$ is low and $K_{d}$ hight the object will be more similar to a water balloon.

To have the equilibrium (Figure $2 \mathbf{a}$ ), the force released by the tendon to the fingertip $\left(F_{n}\right)$ must be equal to the force generated by the virtual object $\left(F_{m}\right)$.

Because of we have to comply with the bandwidth of the virtual object, we need to perform the calculation of $F_{n}$ in real time; indeed any delay can make the system unstable and therefore unusable for our purposes (for more details see section 4.4).

\subsection{Electro-cutaneous stimulation system}

The touch sensation during the interaction with the virtual object is obtained through an electrode fixed between the glove and the user's fingertip (see figure 3 ). When the virtual finger collides with the virtual object the electrode is activated in order to evoke a touch sensation.

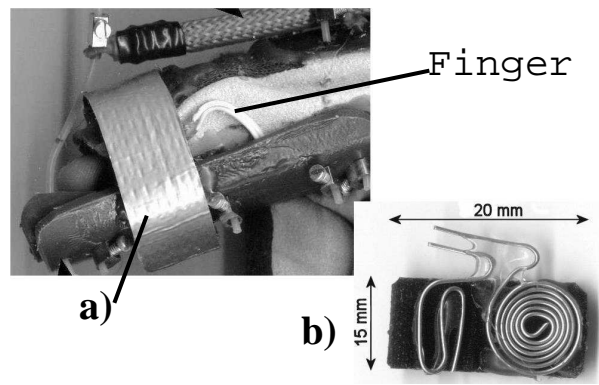

Figure 3: a) Electrode position b) Electrode shape.

In accordance with TENS (Transcutaneous Electric Nerve Stimulation) theory the current injected into the human tissue causes the depolarization of the semipermeable membrane of the skin's receptors and therefore the generation of action potentials that are interpreted by the central nervous system like real sensorial stimuli. 
Using a controlled TENS stimulation, we can therefore evoke touch sensations by recreating the "correct" sequence of action potentials along the axon fibers. In theory, if it would be possible to stimulate the single receptor or nerve, we will be able to generate any kind of touch sensation. Unfortunately this is not easy to obtain for many reasons, the most important being:

1. The electrode current interests many receptors at the same time

2. We do not have the proper technology to build array of electrodes with a resolution comparable with the receptors acuity

3. During the stimulation many kind of receptors are interested by the injected current.

4. It is very difficult, with superficial electrodes, discriminate different skin depths.

To deal with the first two issues it is necessary to increase the resolution of the tactile display; this means that we have to use a matrix of electrodes instead of a single one, as we are currently studying and whose preliminary results are reported in section 4.3. Due to the other two issues, the touch sensations we can recreate are very simple ( more detail in section 5 ).

In order to understand how to design an efficient and efficacious TENS stimulator it is very important to model, from the electrical point of view, the skin and the contact between the electrode and the skin.

In their work Kajimoto H. et al [8] described the equivalent electric membrane model. They related the potential value of the membrane surface with the corresponding inner value for the impulsive stimulus, according to the Hodgkin and Huxley theory. In first approximation, the selectively permeable cell membrane acts as an ohmic resistance that opposes itself to the free movement of ions through the membrane. Even if the cell membrane is fully impermeable to specific ions, the variation of the potential causes, experimentally, a displacement current due to the presence of some lipid layers into the membrane.

The electrical potential of a generic point of the external membrane $P(x, t)$ may be induced by dipping the nervous axons into an electrical field. The current density value, in the generic point $i(x, y, t)$ of the epidermic tissue, can be described by equation 2 , according to the electric schema shown in figure 4 .

$$
i(x, y, t)=\frac{I(t)}{2 \cdot \pi \cdot R}
$$

where $R$ is the distance between the generic point into the tissue, and the electrode set on the skin surface. It is calculated as $R=\sqrt{x^{2}+y^{2}}$. The generated potential $P$, due to the electric field $\vec{E}$ produced by the skin-electrode, can be modelled as in equation 3 , where $I(t)$ is the current injected directly into the skin, and $\rho$ is the skin resistivity, typical of each subject. In a generic point 


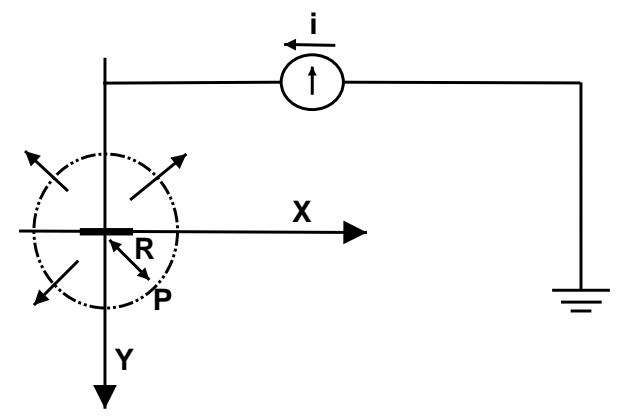

Figure 4: Electric field generated by a punctual electrode model.

of the external surface of the semipermeable membrane the potential value is imposed by the injected current to the $P$ value.

$$
P(x, y, t)=\frac{\rho \cdot I(t) \cdot \log (R)}{2 \cdot R}
$$

The skin-electrode contact model can not be approximated to a short circuit due to the contact resistance that produces valuable reduction phenomena. In addition to the specific tissue resistivity $\rho$ we have to consider the contact resistance between the electrode and the skin surface. In first approximation [21], we can model the skin-electrode contact as shown in figure 5 .

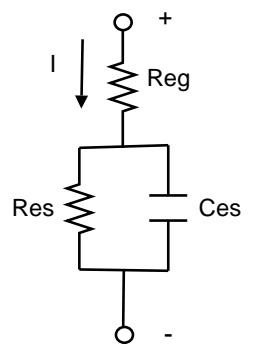

Figure 5: Electrode schema.

$R_{e g}$ is the resistance between the electrode and the skin surface, $R_{e s}$ and $C_{e s}$ are the resistance and the capacity of the electrode-skin interface. According to previous works and empiric tests [21], $R_{e g}$ results smaller than $R_{e s}$ and can be ignored in a first approximation. Therefore, if $V_{e s}$ is the impulse amplitude applied to the electrode-skin interface, we can write the tension value present on the subject tissue $V_{p p}$ as in equation 4 and 5 .

$$
V_{p p}(t)=V_{e s} \cdot\left(1-e^{-\frac{t}{\tau}}\right)
$$




$$
V_{p p}(t)=V_{e s} \cdot e^{-\frac{t}{\tau}}
$$

Furthermore, it is possible to increase the skin-electrode contact quality using a conductive gel that reduces $R_{e g}$. With this model we can change the membrane generator potential $V(t)$ varying the injected current $I(t)$ and taking into account the specific resistance and capacitance of each subject's skin.

In our glove the electrode is controlled by a custom built TENS (Transcutaneous Electrical Nerve Stimulation)-board that generates a generic biphasic wave with a frequencies range between $1 \mathrm{~Hz}$ and $5 \mathrm{KHz}$ and current intensity between 0 and $10 \mathrm{~mA}$. The area of the positive pulse is nearly equal to the area of the negative impulse. This is important to avoid that the electrolysis phenomena might cause a permanent tissue damage.

The TENS board is divided into two main blocks. The first block is the wave generator; it works at low tension $(5 \mathrm{~V})$ and interacts with a PCL-812 A/D board through four dedicated channels. Thank to this the TENS-board is completely controlled via software. It is possible to regulate the current injected, making an instantaneous control ring (via software), both for the safety and the adaptability to different users.

The second part of the board amplifies the signal through a tension transformer connected to the electrode. The transformer elevates the voltage from $5 \mathrm{~V}$ to $100 \mathrm{~V}$, high voltage is necessary to inject enough current into the tissue.

\subsection{Waveform features and multi-electrodes system archi- tecture}

Before doing the experiments on the human subjects we tested and verified the glove and the TENS-board. Even if the output current (delivered to the electrode) is hardware limited, and therefore there are not dangers for the user, an incorrect control of it can produce uncomfortable sensations.

To test the system we connected the output channel to a fixed resistive load and measured the tension and the current at different frequencies and duty-cycles.

In figure 6 we can see a first test were we applied the biphasic signal (Volt) on a resistive load of $10 \mathrm{~K} \Omega$, the current was fixed to a value of $10 \mathrm{~mA}$ and the frequency to $100 \mathrm{~Hz}$. The signal on the bottom is the impulsive wave (with an amplitude of $5 \mathrm{~V}$ ) generated by the wave-generator module. The signal on the top is the biphasic wave; the area of the positive impulse is equal to the area of the negative one, in order to avoid the electrolysis phenomena inside the biologic tissue. We can also observe that there is a little magnetization effect at the end of each positive impulse due to the transformer; this is very low because we prevent it with a proper electronic circuit.

In order to govern a matrix of electrode we developed also an 8-channels TENS board that will allow us to generate complex patterns of stimulation. Thanks to this device it will be possible to generate slipping sensations, to evoke the edge detection of the virtual object, and furthermore to evoke different kinds of touch sensations [8]. In figure 7 we see the test of the multi-channels TENS 


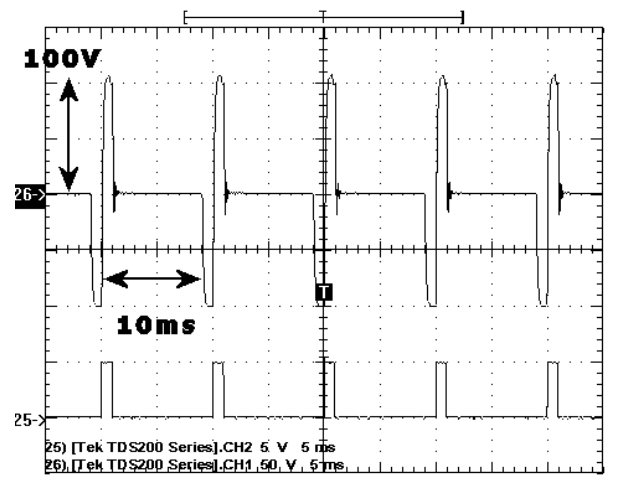

Figure 6: Biphasic wave at $100 \mathrm{~Hz}$ on a resistive load of $10 \mathrm{~K} \Omega$.

board on 8 resistive loads of 100k; each biphasic impulse is delayed relatively to the previous channel of $10 \mathrm{~ms}$. This delay is not perceivable by the user that will feel a concomitant stimulation on all the contact areas with electrodes.

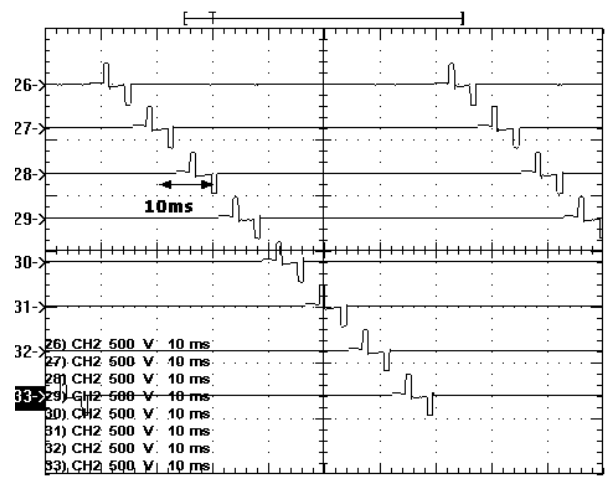

Figure 7: Multi-channels TENS-Board, Biphasic wave at $100 \mathrm{~Hz}$ on a load of $100 K \Omega$

\subsection{Acquisition and control systems}

The following schema (figure 8) presents the whole acquisition and control architecture. Each block is described by a name and its implementation technique (hardware/software).

All the sensor measurements have been normalized and multiplexed using an electronic board and then broadcasted through a single analogical channel to an A/D general purpose card (PCL-812) mounted on a PC executing the xPC-target tool of Matlab. Thanks to xPC-Target architecture we can build physical interfaces and control levels and execute them on different computers, 


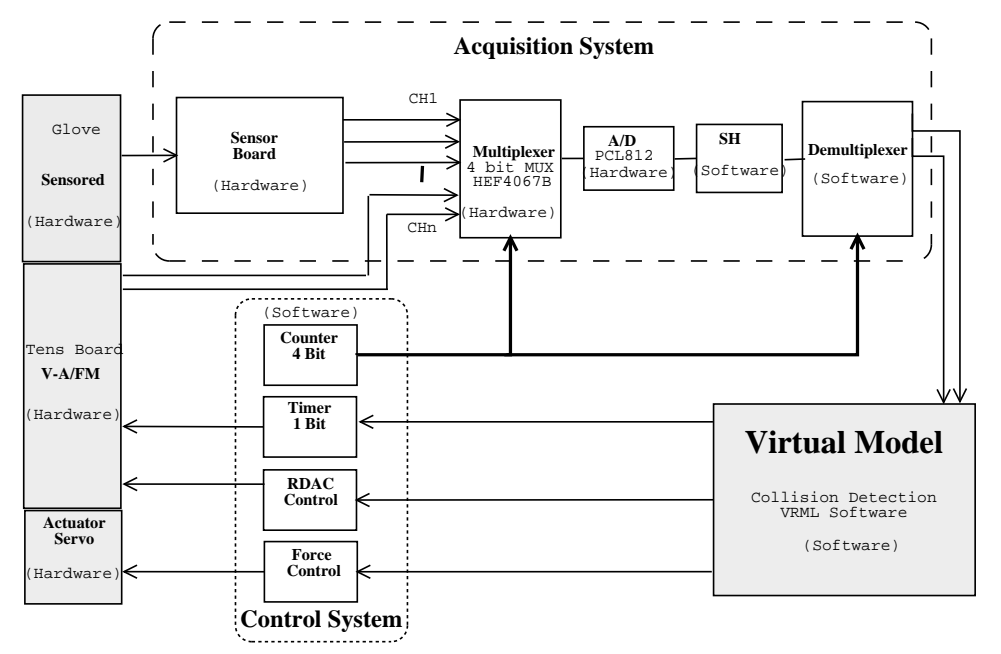

Figure 8: The acquisition and control block of the entire process.

Target and HostPCs. Target-PC plays also the role of implementing a first control loop to determine and generate the real-time value of injected current. A specific value is assigned by the virtual model according to the object surface characteristics; the control module sends data to the TENS-board in order to stabilize that value. This is important to generate similar sensations in different subjects.

Target-Pc is connected, using RS232 interface, with a mobile PC that plays the main role in building the world model. The model is in a VRML file that can be viewed and analyzed by a $\mathrm{C}++$ program with capabilities of collision detection, based on the v-collide algorithm [22]. The virtual model simulator is composed by two main parts: a communication module and an external module. The communication module plays the role of interfacing Matlab with the external VRML module. The external module implements the graphical engine and records all the objects into a tree data base that can be sent and parsed in real-time by the v-collide functions in order to determine collisions between objects.

The Host-PC can realize a second control loop based on angular-sensor measurements, evaluating collisions and then, through the actuator system, binding the finger movement and sending the proper electro-cutaneous stimulation to the finger-tip.

The third and last control loop is made by the user through a visual interface that shows the virtual 3D model and enables control on every process variables.

All the software modules are built in Simulink and compiled for real-time execution in Matlab. A win32 $\mathrm{C}++$ application for 3D model visualization and collision detection is also integrated. 


\section{The qualitative haptic sensation generated}

We experimented our haptic device on 5 volunteers, males and females, with age between 23 and 30 years. Each subject was initially informed about the general purpose of the haptic device and about the scope of the experiment. The subject was asked to sit down in a comfortable position and after was helped to dress the glove. Due to the different hands dimension of each person, a setup and calibration of the system was required. In particular it was possible to adjust the glove's dimension by the regulation of some strip bands located around the wrist and around the fingers, as well as to adjust the electrode location. This was very important to avoid an uncomfortable sensation caused by a bad contact position.

However the subject was not informed about the signal features, in order to do not influence his response. Between two different tests a break of 1 minute was applied, to avoid the receptors adaptation to the stimulation signals. Indeed there is evidence [23] that a persistent electro-tactile stimulation causes the elevation of the sensation threshold and this is principally due to receptors adaptation [24] as well as to a psychophysical adaptation.

During the experiments subjects were asked to describe the sensations perceived. We predefined a closed set of answers for both the kind and the intensity of the sensation felt.

Each experiment was repeated five times with every subject in order to increase the results significance. The experiments were organized in three main phases.

At first we investigated the role of the frequency and current intensity in changing the kind of sensation felt by the subject. The pulse's width, in this case, was maintained constant at half period.

In the second series of experiments we maintained constant the frequency and the current intensity and we varied only the wave duty cycle.

The last series of experiments were done to understand the psychophysics effects due to the combination of a tactile stimulus with a force and visual feedback; we found that stimulating more sensorial channels at the same time increases the realism during the interaction with the virtual environment.

In the following sub-sections we describe in detail each of these experiments and the obtained results.

\subsection{Role played by the stimulation intensity and frequency}

For the first experience, we prepared seven different frequency tests (from $5 \mathrm{~Hz}$ to $400 \mathrm{~Hz}$ ) each differentiated in four levels of current intensity (from low to very high). This means we have a global test set of 28 values for each subject.

The two sets can be described by equation 6 (values for $I_{f}$ are expressed in $\mathrm{Hz}$ ) and equation 7.

$$
I_{f}=\{5,10,20,50,100,200,400\}
$$




$$
I_{i}=\{\text { Low Middle High VeryHigh }\}
$$

Each value belonging to the $I_{i}$ set is defined by the correspondent peak current interval as following:

- $I \epsilon L o w \Leftrightarrow i \leq 1 m a$

- I $\epsilon$ Middle $\Leftrightarrow(i>1 m a) \wedge(i \leq 2.5 m a)$

- $I \epsilon H i g h \Leftrightarrow(i>2.5 m a) \wedge(i \leq 4 m a)$

- IєVeryHigh $\Leftrightarrow i>4 m a$

We set the current injected at fixed values $(1 \mathrm{~mA}, 2.5 \mathrm{~mA}, 4 \mathrm{~mA}$ and $5 \mathrm{~mA})$, but due to hardware limitations, the system needed some time to stabilize the current after a variation of the skin-electrode impedance; this may happen if the fingertip pressure on the electrode changes. Because of this we chose to represent the current intensity using a range of values instead of punctual values. The final test set can be described by the 28 position table described by equation 8 .

$$
I=I_{f} \times I_{I}
$$

For each test the subject explained the sensation felt during the experience. We can state that each sensation, produced by the stimulation of a mechanoreceptor, has two main components: the intensity level and the sensation evoked in the human mind [25]. We therefore prepared two response sets in order to map either components. The first set is composed by six possible intensity response (from NoSesation to Pain); the second one has seven elements corresponding to seven possible sensations. We can write these two sets as in equation9 and equation10.

$$
\begin{gathered}
R_{I}=\{\text { NoSens., Low, Midd., High, Irrit., Pain }\} \\
R_{f}=\{\text { NoSens., Beats, Itch, Vib., Ting., Rasp., Warm }\}
\end{gathered}
$$

In table 1 we report a description of each sensation felt by the subject.

\begin{tabular}{|l|l|}
\hline \hline Feel & Brief Description \\
\hline NoSens. & Any sensation is felt \\
Beats & Repetitive pressure on the fingertip \\
Itch & Itch distritbuted on the contact surface \\
Vibra. & Clear sensation of vibration \\
Tingle & Little vibration, similar to a Tingle \\
Rasp. & Sensation of Rasping \\
Warm & Warming sensation, localized stinging \\
\hline
\end{tabular}

Table 1: $R_{f}$ Brief description of possible touch sensation felt by the subject 
To describe the experiment results we prepared a final response set that results from equation11.

$$
R=R_{f} \times R_{I}
$$

The first experiments can be described as following:

$$
\forall f \epsilon I_{f}, \forall i \epsilon I_{I} \Leftrightarrow \operatorname{Resp}(f, i) \epsilon R
$$

For each couple of values in the frequency-intensity set $\left(I_{f} X I_{I}\right)$ we found a response belonging at the response set $R$.

Figure 9 resumes the results of this first experiment. In each graph we put on the $y$-axis the number of people giving the same response to a certain stimulus. The $\mathbf{S}$ and $\mathbf{I}$ axes represent the sensation intensity felt by the subject and the current intensity of the stimulation signal respectively. The four graphs report data obtained at four different frequencies of stimulation (for brevity we do not represent here all the seven frequencies).
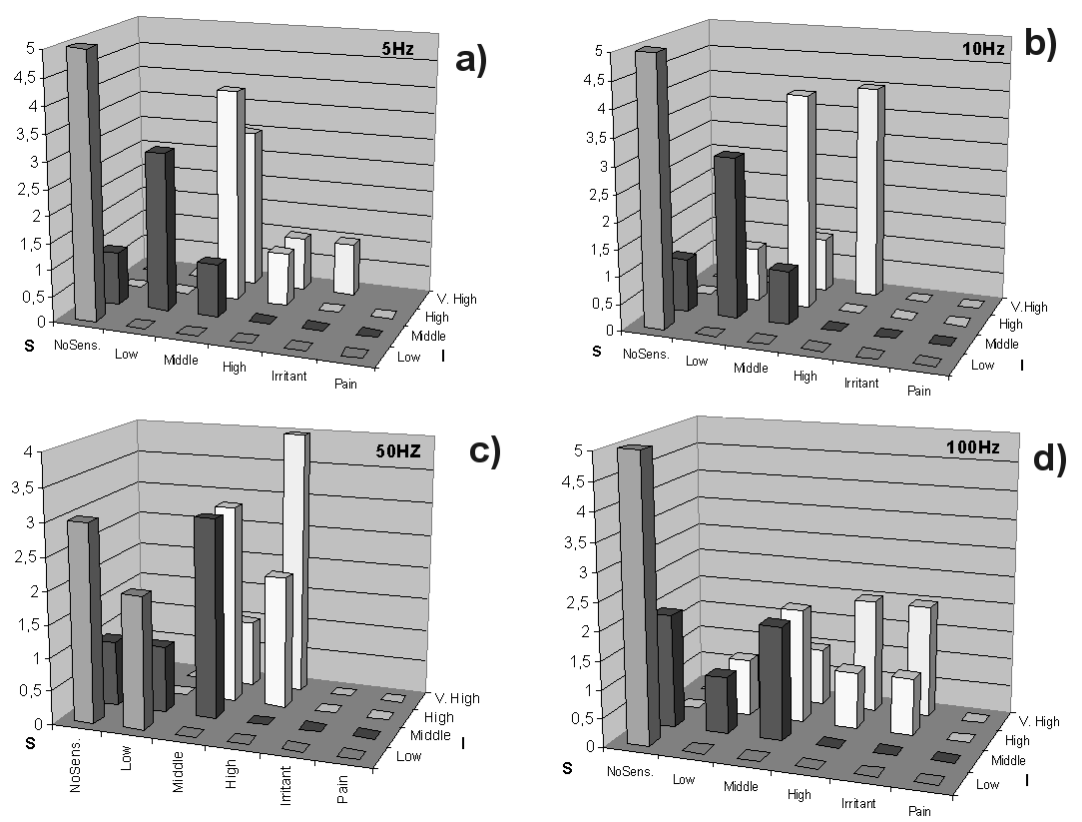

Figure 9: The four graphs represent the responses of five subjects for the sensation felt at different level of current. Each graph represent a different frequency of stimulation

As we see in the graph (a) of picture 9, the current increasing affects the intensity of the sensation felt by the subjects. This is in accordance with the physiology of the receptor, since the more is the current injected in the tissue, the more the membrane depolarizes causing a major frequency of action potentials. 
We note also that an increasing in the stimulation frequency (graphs from a to d) seems to amplify the intensity of the stimulus felt.

From the experiments we found that values of current under 1ma (Low) are inappreciable to most of the subjects. Subjects felt low sensation between $1 \mathrm{ma}$ and $2.5 \mathrm{ma}$ (Middle). At this level they can be distracted by other stimuli, like people speaking. This is an important consequence of filter theory. Values between $2.5 \mathrm{ma}$ and $4 \mathrm{ma}$ (High) are strongly felt by the subjects. In this case subjects cannot be distracted by other external stimulus. Values up to $4 \mathrm{ma}$ (Very-High) are considered strong and uncomfortable. In some, rare case subjects feel pain. The high variance for this data suggests us to increment the number of elements of $I_{i}$ decreasing steps especially for high values $(4 \mathrm{ma}+)$.

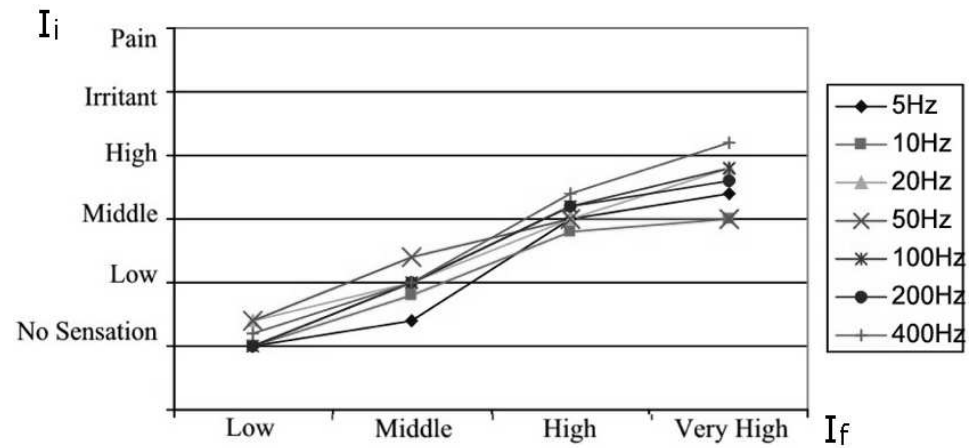

Figure 10: Subject sensations by electric pulse intensity.

To better consider the dependency of the sensation felt from the real intensity of the electrical stimulus we have averaged the data obtained, as illustrated in figure 10, that shows how the sensation perceived by the subject logarithmically grows with stimulation intensity. This supports the Steven's law [26] by which the sensation felt grows following the equation 13.

$$
S=K \cdot I^{b}
$$

where $S$ is the sensation perceived, $I$ is the stimulus entity, $K$ and $b$ are two constants that depend on each subject. In the transcutaneous stimulation $K$ and $b$ depend also on the impulse frequency. This is true if we think that the Hodgkin and Huxley [27] relation, between generator potential and axon activation potentials, suggests a proportionality between frequency of axons potential and stimulus intensity. To study the sensations evoked by the electrical stimulation, we prepared a second double entering table in which we described for each frequency, current $(f, i)$ couple the $R_{f, i}$ element the subject response.

The table in figure 2 shows only the dependency by frequency values of the stimulation signal. The kinds of sensation are the same reported in table 1.

To better interpret this data we can build a graph (Figure 11). 


\begin{tabular}{|c|c|c|c|c|c|c|}
\hline Frequency & B. & I. & V. & T. & R. & W. \\
\hline $5 \mathrm{~Hz}$ & 11 & 1 & 0 & 0 & 0 & 0 \\
$10 \mathrm{~Hz}$ & 11 & 2 & 0 & 0 & 0 & 0 \\
$20 \mathrm{~Hz}$ & 7 & 1 & 5 & 2 & 0 & 0 \\
$50 \mathrm{~Hz}$ & 2 & 5 & 7 & 2 & 0 & 0 \\
$100 \mathrm{~Hz}$ & 0 & 1 & 8 & 1 & 3 & 0 \\
$200 \mathrm{~Hz}$ & 0 & 6 & 7 & 1 & 0 & 0 \\
$400 \mathrm{~Hz}$ & 1 & 2 & 6 & 1 & 0 & 4 \\
\hline
\end{tabular}

Table 2: Subject sensations by frequency values.

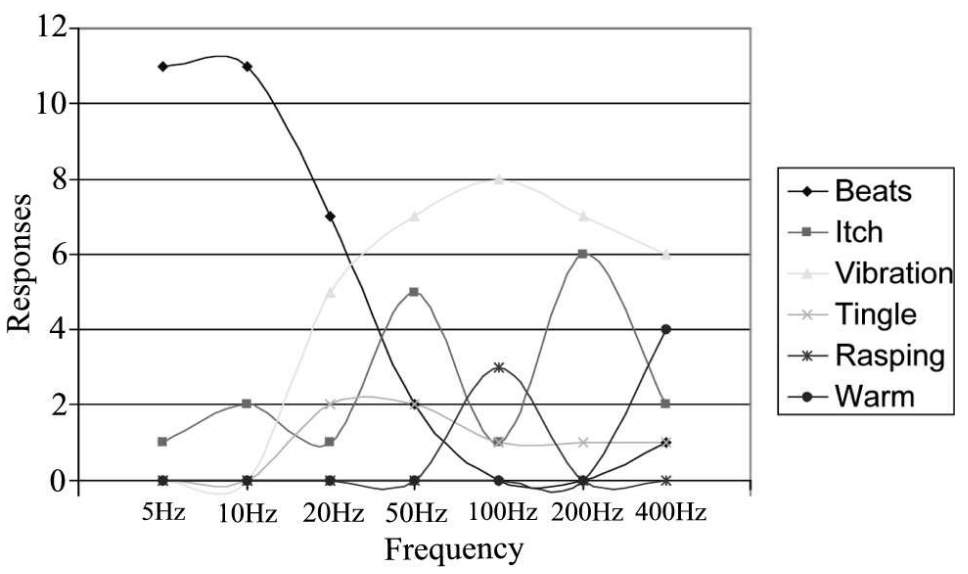

Figure 11: Data graph of subject sensations by frequency values.

We can see that at very low frequencies (from $5 \mathrm{~Hz}$ to $25 \mathrm{~Hz}$ ) the principal sensations felt are beats and small pressure. Merkel cells are sensible to that frequency and seems to be specialized in detection of pressure and surface deformations. At middle frequency (from $100 \mathrm{~Hz}$ to $200 \mathrm{~Hz}$ ) a new sensation of vibration was evoked. Users can't understand, in many cases, the period of the impulsive current but can only feel a sensation of rapid vibration or grasping object under the fingertip surface. If we think that grasping sensation can be related to hard vibration, we can assure that the $82 \%$ of the sensations evoked by a stimulus of about $100-200 \mathrm{~Hz}$ can be identified as a vibration stimulus. This agrees with former findings that suggested that Pacinian Corpuscles are sensible to vibration and operate at that frequency [28].

\subsection{Role played by the duty-cycle of the wave}

In the second experiment we tried to understand the role played by the impulse Duty-Cycle of the electrical stimulation wave. We fixed the frequency at two significant values $(10 \mathrm{~Hz}-50 \mathrm{~Hz})$ and the current at two intensity levels. Then we asked the subject to describe the difference felt while varying pulse width 
from $10 \%$ to $90 \%$ of the whole period.

Question sets are described by equation 14, equation 15 and equation 16

$$
\begin{gathered}
I_{f}=\left\{\begin{array}{ll}
10 \mathrm{~Hz} & 50 \mathrm{~Hz}
\end{array}\right\} \\
I_{i}=\left\{\begin{array}{ll}
\text { Middle High }
\end{array}\right\} \\
I_{w}=\left\{\begin{array}{ll}
10 \% & 90 \%
\end{array}\right\}
\end{gathered}
$$

where $I_{f}$ describes test frequency values, $I_{i}$ is the intensity level set and $I_{w}$ is the wave duty-cycle of the experiment. Response set $\mathbf{R}_{\mathbf{w}}$ is described by table 3.

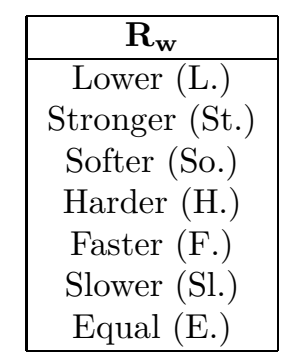

Table 3: Response set

The Lower and Stronger values means that the subject feels the same sensation but perceives some variation in the intensity level. The Softer and Harder values means that subject feels the same intensity of the half width impulse but with a less or more clear sensation. The Faster and Slower values are connected to the perceived sensation of changed speed. Finally Equal value means that the subject doesn't perceive any kind of variation.

The whole experience can be described by equation 17 .

$$
\forall f \epsilon I_{f}, \forall i \epsilon I_{i}, \forall a \epsilon I_{w} \Leftrightarrow \operatorname{Resp}(f, i, a) \epsilon R_{w}
$$

We applied four tests for each single subject, therefore we recorded a total of 20 experiences. Results are reported in table 4, where we grouped data by impulse width.

\begin{tabular}{|c|c|c|c|c|c|c|c|}
\hline a & L. & St. & So. & H. & F. & Sl. & E. \\
\hline $10 \%$ & 11 & 0 & 0 & 4 & 0 & 0 & 5 \\
$90 \%$ & 0 & 9 & 8 & 0 & 1 & 0 & 2 \\
\hline
\end{tabular}

Table 4: Subject responses about sensation driven by pulse width modulation.

Subjects feel lower sensation (Lower) for small duty-cylce (10\%) but also a clear sensation was evoked (Harder). For greater duty-cycle (90\%) the subjects feel stronger sensation (Stronger) but smoother (Softer) than the first one. 
We can use pulse width modulation in order to evoke clear or smooth tapping sensation based on the same frequency level.

\subsection{Integration of tactile, force and visual feedbacks}

The last experimentation involved the force feedback, the cutaneous touch generator and the visual feedback all together. During these experiments the entire system is employed; if a contact is reached, the servo reacts opposing the finger movement. It is possible to change the servo speed and the touch position in order to simulate hard and soft objects of different dimensions.

Initially we applied only the force feedbacks. The user was constricted by the glove structure to move only the index finger. He was allowed to make repeated finger movements of flexion and extension in order to explore the virtual object. During this phase only the force sensation was activated, so the subject didn't feel clearly the initial contact with the object.

In the second experiment, also the tactile sensation was recreated; as before the person was asked to describe the sensation and compare it with the first one. The results of this experiment are reported in table 5 .

Table 5: Experiences touching the virtual object

\begin{tabular}{|c|c|c||l|}
\hline$K_{e}\left[\frac{N}{m m}\right]$ & $K_{d}\left[\frac{N}{\frac{m m}{s}}\right]$ & Touch & Sensation \\
\hline \hline 0.1 & 0 & NO & $\begin{array}{l}\text { Push a rubber } \\
\text { object with the } \\
\text { entire finger }\end{array}$ \\
\hline 0.1 & 0 & YES & $\begin{array}{l}\text { Push a } \\
\text { rubber object } \\
\text { principally with } \\
\text { the fingertip }\end{array}$ \\
\hline 0 & 0.1 & NO & $\begin{array}{l}\text { Moving the } \\
\text { entire finger } \\
\text { in a liquid }\end{array}$ \\
\hline 0 & 0.1 & YES & $\begin{array}{l}\text { Moving the } \\
\text { fingertip in } \\
\text { a liquid }\end{array}$ \\
\hline \hline
\end{tabular}

What is clear from these data is that also a simple vibration, instead of a real touch sensation, can improve the localization of the contact force with the virtual object.

We can also modulate the frequency to give a more complete touch information. For example as a possible law we can use equation 18; in this case the frequency is proportional to the contact force.

$$
f=\operatorname{sat}\left(K \cdot F_{V}\right)
$$




$$
\operatorname{sat}(x)= \begin{cases}x & \text { if } x \leq T h \\ f_{\max } & \text { if } x>T h .\end{cases}
$$

When the user finger starts to touch the virtual object the force $F_{V}$ is low and so the vibration's frequency $f$, but when the contact force increases also the frequency rises proportionally to it. In equation 18 the term sat is a saturation function that permits to fix a maximum frequency and to avoid uncomfortable sensations for the user.

In a second phase we activated also the visual feedback. We presented to the subject a VRML virtual model of the human hand and of the objects (Figure 12). In this experiment we maintained constant the intensity and frequency of the electro-tactile stimulation. We introduced an electrical stimulation $(100 \mathrm{~Hz}$, middle intensity level) on the fingertip when the subject reached the virtual object.

In this case when the system detects a collision the force feedback and the cutaneous stimulation are activated in order to give the subject a multi-modal sensation.
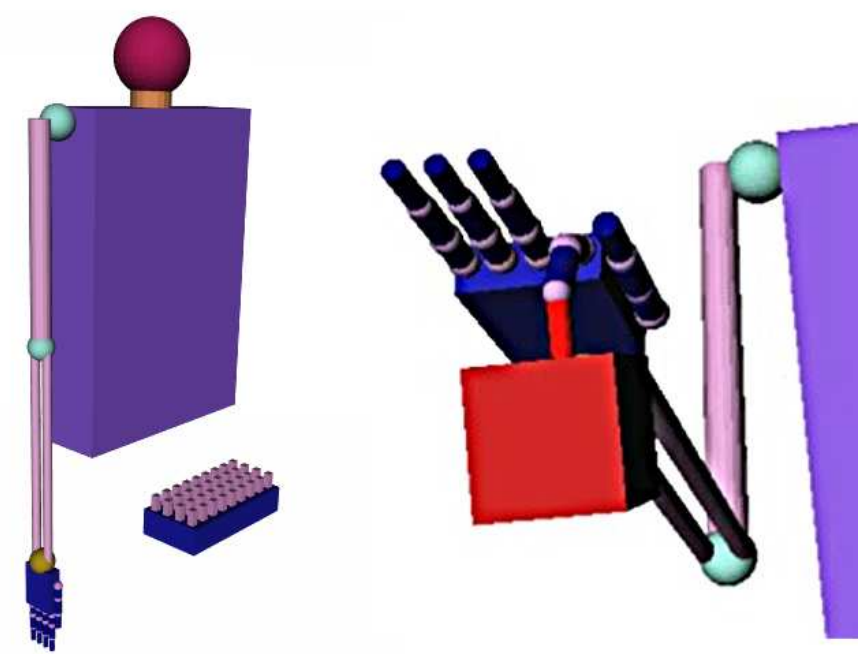

Figure 12: The virtual model as presented on the screen.

For all these experiments we used two virtual objects of different dimensions. For each object we tested two different materials, hard and soft. We can describe the question set related to the object dimension through equation20

$$
I_{d}=\{\text { Small Big }\}
$$

and the question set related to the object hardness through equation 21

$$
I_{f}=\{\text { Soft Hard }\}
$$


the resulting question set is composed by the 4 positions table described in equation 22

$$
I_{n}=I_{d} \times I_{f}
$$

where $n$ is the experiment number (from 1 to 3 ). For each part of this experiment, we presented the virtual object to the subject and then we asked him to recognize its properties choosing into a response set of the same kind of $I_{n}$.

Subjects recognized object hardness and dimension in each phase, but only when we introduced visual system, they were able to assign also a correct shape interpretation for the touched object.

Summarizing, with only the force feedback subjects feel the consistency of the object, they can distinguish between hard and soft objects. Combining force feedback and electrical touch subjects can accurately determine when the contact with object begins, but still they do not feel a realistic touch sensation. When the whole system was tested subjects easily affirm that they were touching an object of the correct shape and material.

This is an important result if we think that in the present system there is a severe hardware limitation. Indeed the introduction of the visual system produces a delay into the frame rate of about $110 \mathrm{~ms}(100 \mathrm{~ms}$ due to the $\mathrm{V}$ Collide system and $10 \mathrm{~ms}$ due to the Simulink model), five times higher than the servo impulse ratio. This delay is principally due to the algorithm for collision detection and also to the time required to permit the communication between different software modules.

Another possible improvement of the system is using an array of electrodes (see section 4.3) instead of a single electrode. Controlling independently each single electrode in current intensity and frequency will make it possible to generate more complex stimuli. For example, properly activating the electrodes, the user moving the finger on the virtual object surface would know the full object dimensions and shape, not only one dimension. Furthermore, controlling the electrodes current independently, it will be possible to generate different kinds of pattern of stimulations and therefore to selectively activate different kinds of mechano-receptors [8], evoking a wider range of tactile sensations and therefore improving the interaction realism.

\section{Conclusions and future work}

Haptic Interfaces are those systems, composed both of hardware and software, that permit to expand the media of communication between man and machine, adding an entirely new communication channel: the feeling of physical contact. Looking more in detail, we have presented a virtual environment (developed in VRML) that runs as a simulation on a machine. This environment is translated to phenomenons, perceptible by humans, by the interface we developed. Consequently these stimuli are sensed and interpreted by the conscious human being and becomes visual, and tactual experiences. 
Normally Haptic interfaces are used in both directions: they are input and output devices at the same time. Despite the fact that the same physical object (the glove in our case) implements an input and an output device at the same time, it must be clear the distinction between the functions performed by these two components. In this scenario there are two closed control loops: one that connects the human to the virtual environment, and the other that connects the remote controlled machine to the virtual environment.

We developed an innovative haptic device for applications in the field of Virtual Reality and Robotics. We proposed a new architecture that combines a two-channels TENS stimulator fully digitally controllable, a glove interface equipped with sensors, actuators and electrode on the index fingertip, and a PC based control system that permits the interaction of the user with a simple virtual object. The software allows to set the object parameters and to control the electro-tactile stimulator.

We conducted experiments to understand the correlations between the properties of a tactile stimulation signal and the kind and intensity of the sensation felt by the subject. We found that increasing the current intensity and impulse width effects the strength of the sensation perceived by the subject. From our data it seems also clear that the frequency covers an important role in influencing this feeling.

In comparison with other haptic devices [2], [3], we exploit the electro-tactile stimulation in order to recreate the sensation of contact during the interaction with the virtual object. In comparison with other studies [6], [7], [4] we tried to combine different type of sensorial stimulus with the main goal to increase the realism of the interaction with the virtual object.

\section{References}

[1] Heller Morton A. Tactile picture perception in sighted and blind people. Behavioural Brain Research Volume, 135(1-2):65-68, September 2002.

[2] Asamura Naoya, Tomori Naruyuki, and Shinoda Hiroyuky. A tactile feeling display based on selective stimulation to skin receptors. Proc. IEEE Virtual Reality Annual International Symposium, March 1998.

[3] Caldwell Darwin G., Tsagarakis N., and Giesler C. Integrated tactile/shear feedback array for stimulation of finger mechanoreceptor. Proc. IEEE International Conference on Robotics and Automation, 1:287-292, 1999.

[4] Yoshikawa T. and Nagura A. A touch force display system for haptic interface. Presence: Teleoperators and Virtual Environments, 10(2):225235, April 2001.

[5] Kaczmarek Kurt A. and Webster John G. Voltage-current characteristics of the electrotactile skin.electrode interface. IEEE Engineering in Medicine and Biology Society 11TH Annual International Conference, 1989. 
[6] Kaczmarek Kurt A. and Haase Steven J. Pattern identification as a function of stimulation current on a fingertip-scanned electrotactile display. IEEE Transaction On Neural System And Rehabilitation Engineering, 11(3):269275, September 2003.

[7] Kajimoto Hiroyuki, Kawakami Naoki, Tachi Susumu, and Inami M. Smarttouch: electric skin to touch the untouchable. IEEE Computer Graphics and Applications, 24(1):36-43, January-February 2004.

[8] Hiroyuki Kajimoto, Naoki Kawakami, Taro Maeda, and Susumu Tachi. Tactile feeling display using functional electrical stimulation. In Proc. ICAT'99, 1999.

[9] Poletto Christopher J. and Van Doren Clayton L. A high voltage, constant current stimulator for electrocutaneous stimulation through small electrodes. IEEE Transaction on Biomedical Engineering, 46(8):929-936, August 1999.

[10] Perez C.A., Holzmann C.A., and Osorio M.E. Optimization of the envelope of a short duty cycle pulse waveform for tactile stimulation. IEEE Proc. Engineering in Medicine and Biology Society 17th Annual Conference, 2:1137-1138, September 1995.

[11] Salisbury Kenneth J. Making graphics physically tangible. Communications of the ACM, 42(8):75-81, August 1999.

[12] Ruspini Diego and Khatib Oussama. A framework for multi-contact multibody dinamic simulation and haptic display. International Conference on Intelligent Robots and Systems, 2000.

[13] Michele Folgheraiter, Ilario Baragiola, and Giuseppina Gini. Teaching grasping to a humanoid hand as a generalization of human grasping data. Lecture Notes in Computer Science, 3303 / 2004:139.

[14] William R. Mark, Scott C. Randolph, Mark Finch, James M. Van Verth, and Russell M. Taylor II. Adding force feedback to graphics systems issues and solutions. In Proceedings of SIGGRAPH 96, Computer Graphics Proceedings, Annual Conference Series, pages 447-452, New Orleans, Louisiana, August 1996. ACM SIGGRAPH / Addison Wesley. ISBN 0201-94800-1.

[15] Richard J. Adams and Blake Hannaford. Stable haptic interaction with virtual environments. IEEE Transactions on Robotics and Automation, 15(3):465-474, 1999.

[16] Blake Hannaford and Jee-Hwan Ryu. Time domain passivity control of haptic interfaces. IEEE Transactions on Robotics and Automation, 18:110, February 2002. 
[17] Kenneth O. Johnson, Takashi Yoshioka, and Francisco Vega-Bermudez. Tactile functions of mechanoreceptive afferents innervating the hand. Journal of Clinical Neurophysiology, 17(6):539-558, November 2000.

[18] H. E. Wheat, L. M. Salo, and A. W. Goodwin. Human ability to scale and discriminate forces typical of those occurring during grasp and manipulation. The Journal of Neuroscience, 24(13):3394-3401, March 2004.

[19] A.J. Brisben, S.S.Hsiao, and K.O. Johnson. Detection of vibration transmitted through an object grasped in the hand. Journal of Neurophysiology, 81:1548-1558, April 1999.

[20] DF. Collins and A. Prochazka. Movement illusions evoked by ensemble cutaneous input from the dorsum of the human hand. The Journal of Physiology, 496(3):857-871, 1996.

[21] Kaczmarek Kurt A. and Webster John G. Voltage-current characteristics of the electrotactile skin-electrode interface. IEEE Proceeding Annual Internetional Conference Eng. Med. Biol. Soc., 11, 1989.

[22] T. Hudson, M. Lin, J. Cohen, S. Gottschalk, and D. Manocha. V-collide: Accelerated collision detection for vrml. Appeared in Proc. of VRML ' 97 , 1997.

[23] Kaczmarek Kurt A. Electrotactile adaptation on the abdomen: Preliminary results. EEEE Transaction On Rehabilitation Engineering, 8(4):499-505, December 2000.

[24] J. F. Hahn. Tactile adaptation in The Skin Senses. C. C. Thomas D. R Kenshalo Ed. Springfield, 1968.

[25] Paul Bach-Y-Rita, Mitchell E. Tyler, and Kurt A. Kaczmarek. Seeing with the brain. Internationa Journal of Human-Computer Interaction, 15(2), 2003.

[26] Stevens SS. Neural events and the psychophysical law. Science, 170(962):1043-50, December 1970.

[27] Hodgkin Alan L. and Huxley Andrew F. A quantitative description of membrane current and its application to conduction and excitation in nerve. Journal of Physiology, 117(4):500-544, 1952.

[28] Kajimoto Hiroyuki, Kawakami Naoki, Maeda Taro, and Tachi Susumu. Tactile feeling display using functional electrical stimulation. In Proc. ICAT'99, 1999. 